Case Report

\title{
Treatment of Skeletal Class III and Anterior Open Bite Using a Multibracket Sys- tem Combined with Oral Myofunctional Therapy
}

\author{
Shinichi Negishi, Takemi Goseki, Sho Kuroe, Kota Sato, and Kazutaka Kasai \\ Department of Orthodontics, Nihon University School of Dentistry at Matsudo, Matsudo, Chiba 271-8587, Japan
}

\section{Article History}

Received 9 March 2021

Accepted 28 April 2021

Keywords :

Skeletal Class III, open bite, myofunctional therapy,

oral function

\begin{abstract}
We present a case of Skeletal Class III and anterior open bite that we treated with a multibracket system and myofunctional therapy to improve oral function. Our aim is to present a case with a treatment that benefits the long-term retention with class 3 intermaxillary elastic and myofunctional therapy. A woman aged 22 years 2 months presented with a chief complaint of difficulty eating due to an anterior open bite. The frontal view of the face was symmetrical, and the lateral view was a straight type. The molar relationship was Angle Class III. Overjet and overbite were $+2.5 \mathrm{~mm}$ and -2.5 $\mathrm{mm}$, respectively. The arch length discrepancy was $-1.0 \mathrm{~mm}$ (upper) and $-0.5 \mathrm{~mm}$ (lower), respectively. The treatment plan was a non-extraction multibracket system with Class III intermaxillary elastic. During her treatment, oral function was improved by myofunctional therapy. On the left side maxillary, the second molar was extracted because of root resorption to erupt third molar. The third molar was then used as the second molar. As a result, her anterior open bite improved, and stable occlusion was achieved over the long term.
\end{abstract}

\section{Introduction}

In the adult orthodontic treatment of an anterior open bite, it is important to assess oral function. Relapse can occur if myofunctional therapy is performed without an understanding of the proper function of the tongue(1). We present a case with a V-shape maxillary dental arch and anterior open bite with a low set of tongue and tongue thrust (2). We obtained good results from treatment with a multibracket system, maxillary Class III elastics, and myofunctional therapy. Oral function such as occlusal force, lip closure force, and tongue pressure improved by myofunctional therapy. This relief was maintained during the retention period. Here, we report that our case achieved individual normal occlusion with longterm stable by proper orthodontic treatment and myofunctional therapy.

\section{Correspondence to :}

Shinichi Negishi

E-mail : negishi.shinichi@gmail.com

doi : 10.5466/ijoms.20.68

\section{Case Description}

A woman aged 22 years and 2 months presented with a chief complaint of difficulty eating due to an anterior open bite. The frontal view of the face was symmetrical, and the lateral view was a straight type(Fig. 1). There was an anterior open bite, and the root of the maxillary left second molar was absorbed. The molar relationship was Angle Class III; overjet and overbite were $+2.5 \mathrm{~mm}$ and $-2.5 \mathrm{~mm}$, respectively. Arch length discrepancies were $-1.0 \mathrm{~mm}$ (upper) and $-0.5 \mathrm{~mm}$ (lower), respectively (Fig. 2). There was a cross bite on the left buccal segment. Cephalometric and panoramic radiographs are shown in Figs. 3 and 4. The lateral cephalometric analysis showed skeletal Class III pattern $\left(\mathrm{ANB} 0^{\circ}\right)$, upper incisor labial inclination and lower incisor lingual inclination $(\mathrm{FH}$ to $\mathrm{U} 1123.5^{\circ}$, FMIA $67.0^{\circ}$ ), and no vertical problem(FMA $23.0^{\circ}$, Gonial angle $123.0^{\circ}$ ) (Table 1). Additionally, the patient had oral habit such as tongue thrust and low position tongue. The values of oral function were occlusal force $379 \mathrm{~N}$, lip closure force $5.0 \mathrm{~N}$, and tongue pressure $36.2 \mathrm{kPa}($ Table 2). 


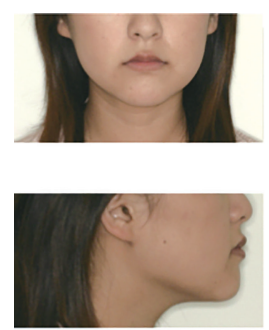

Pretreatment
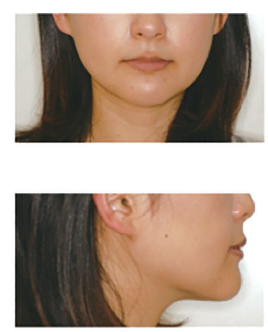

Posttreatment
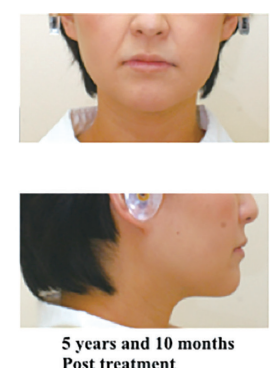

Post treatment

Fig. 1. Facial photographs

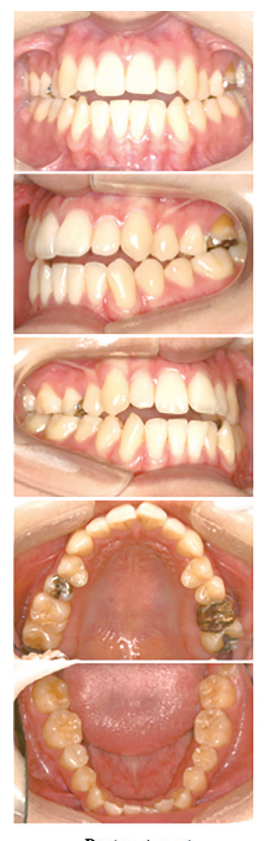

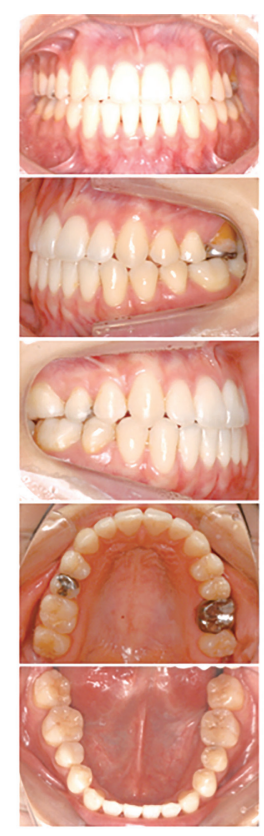

Posttreatment
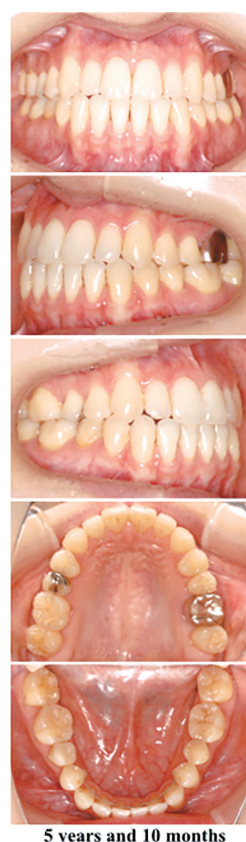

5 years and 10 months

Fig. 2. Intraoral photographs

\section{Diagnosis}

Skeletal Class III, Angle Class III, Anterior open bite

\section{Treatment Objectives}

Our goals were to improve patients' profiles and to provide a harmonious occlusion. These treatment objectives included improving anterior open bite and narrow upper dental arch, establishing an angle Class I relationship, improving tongue function by myofunctional therapy, and long-term stability. A multibracket system was used to achieve the treatment objectives, and a quad-helix appliance was used for expansion of upper dental arch. The reason why we didn't choice orthodontic surgery was the maxillary dental arch can be expansion and the mandibular buccal segment with mesial tipping could be improved by Class III intermaxillary elastic.

\section{Treatment Progress and Results}

The treatment period was 2 years 6 months.

The upper left second molar was extracted for root resorption, then to await the eruption of the third molar. Brackets (.022" straight type) were bonded from 17 to 27 in the maxilla, from 33 to 37 , and from 43 to 47, except for the mandible anterior teeth, and a quad-helix was placed in the palate. A 0.014 -inch nickel-titanium(NiTi) wire was placed in both arches to begin decrowding. A quad-helix was placed in palate to expand the maxilla dental arch. We began myofunctional therapy, which includes specific exercises, to eliminate oral habits such tongue thrusts, that strengthen the facial muscles. Myofunctional therapy was continued until the end of orthodontic treatment, and examination of oral function was performed during the retention period. If the oral func- 

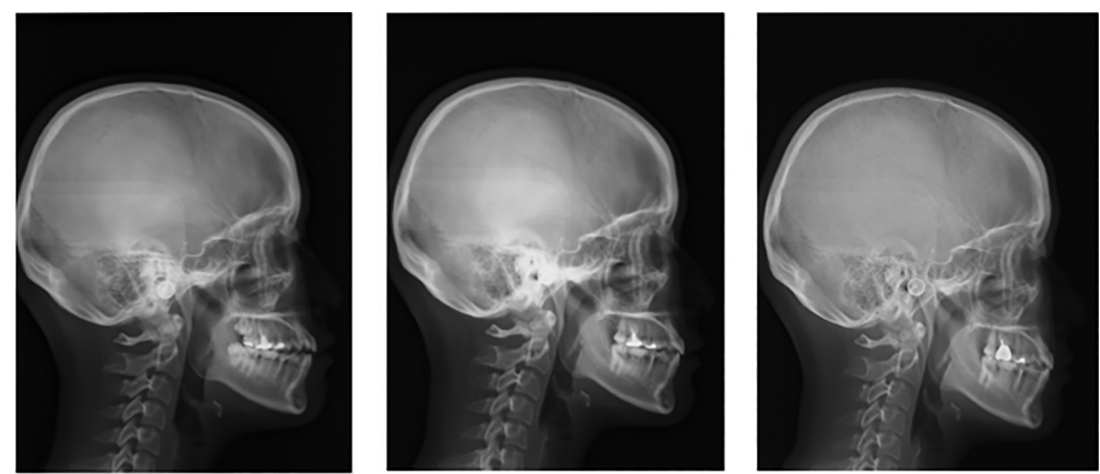

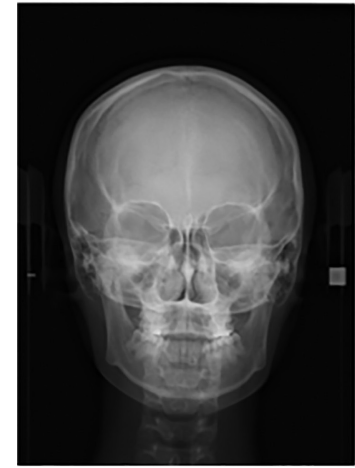

Pretreatment

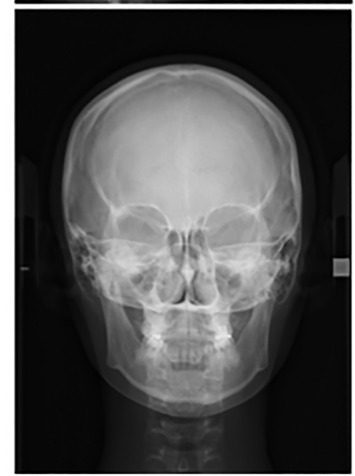

Posttreatment

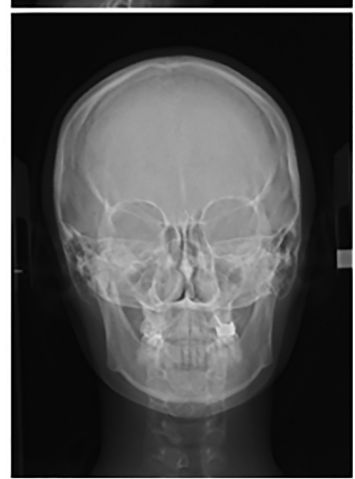

5 years and 10 months Post treatment

Fig. 3. Cepharometric radiographs (Lateral and Frontal)

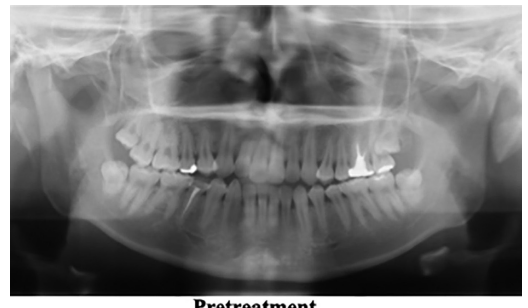

Pretreatment

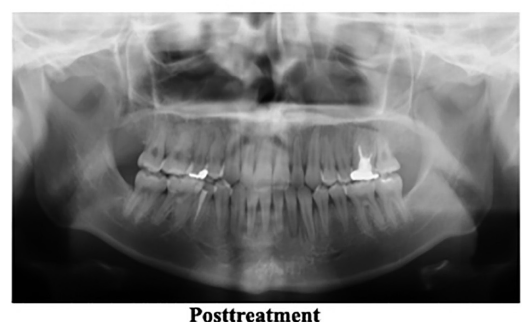

Posttreatment

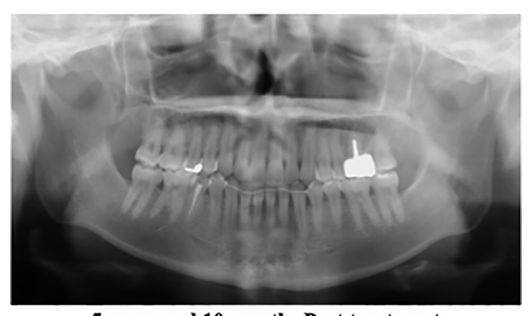

5 years and 10 months Post treatment

Fig. 4. Panoramic radiographs tion was decreased, myofunctional therapy was planned to be performed even during the retention period. The bracket was bonded in the left third molar in the maxilla after recognizing the eruption. A $0.016 " \times 0.022$ " stainless steel(SS) wire was placed in both arches with Class III intermaxillary elastic for distal tipping of mandible buccal segment. The quad-helix was changed to the transpalatal arch to prevent extrusion of the upper first molar. Since space was created in the mesial part of the buccal segment by Class III intermaxillary elastic, the brackets were bonded to the mandible's anterior teeth. A 0.014" NiTi wire was placed and leveling was started. A $0.016 "$ $\times 0.022$ " TMA wire was placed in both arches for detailing. The orthodontic appliances were removed. A circumferential removal retainer and a 0.0175 -inch multistranded stainless steel lingual fixed retainer were attached from the first premolar to the opposite first premolar, then fitted into both arches for retention. The retention period was two years (Begg type in maxillary and canine to canine lingual bar in mandibular), and an examination was performed 5 years and 10 months after removing the orthodontic devices.

Summarizing the treatment results, there was established good anterior-posterior occlusion. FMIA inclined 
Table 1. Cephalometric measurements

\begin{tabular}{lcccc}
\hline & Pretreatment & Postreatment & $\begin{array}{c}\text { 5 years and 10 months } \\
\text { Post treatment }\end{array}$ & Mean \pm S.D. \\
\hline SNA & $81.5^{\circ}$ & $81.5^{\circ}$ & $81.5^{\circ}$ & $81.3^{\circ} \pm 2.7^{\circ}$ \\
SNB & $81.5^{\circ}$ & $81.0^{\circ}$ & $81.0^{\circ}$ & $78.8^{\circ} \pm 2.7^{\circ}$ \\
ANB & $0.0^{\circ}$ & $0.5^{\circ}$ & $0.5^{\circ}$ & $2.7^{\circ} \pm 1.0^{\circ}$ \\
FMA & $23.0^{\circ}$ & $23.0^{\circ}$ & $23.0^{\circ}$ & $26.3^{\circ} \pm 4.0^{\circ}$ \\
IMPA & $90.0^{\circ}$ & $88.0^{\circ}$ & $88.0^{\circ}$ & $96.8^{\circ} \pm 6.4^{\circ}$ \\
FMIA & $67.0^{\circ}$ & $69.0^{\circ}$ & $69.0^{\circ}$ & $56.9^{\circ} \pm 6.4^{\circ}$ \\
FH to U1 & $123.5^{\circ}$ & $116.5^{\circ}$ & $116.5^{\circ}$ & $112.1^{\circ} \pm 5.7^{\circ}$ \\
Interincisal Angle & $123.5^{\circ}$ & $132.5^{\circ}$ & $132.5^{\circ}$ & $123.5^{\circ} \pm 5.5^{\circ}$ \\
Gonial Angle & $123.0^{\circ}$ & $123.0^{\circ}$ & $123.0^{\circ}$ & $118.5^{\circ} \pm 6.5^{\circ}$ \\
SN to Occlusal & $17.5^{\circ}$ & $15.0^{\circ}$ & $15.0^{\circ}$ & $10.0^{\circ} \pm 5.5^{\circ}$ \\
\hline a For Japanese mean (Iwasawa T, Nakakuni M, Matsumoto Y: The profile of normal occlusion and malocclusion \\
(Angl's Class I, II, III) .The journal of Japan Orthodontic Society, 28: 105-112,1969.)
\end{tabular}

forward to lingual from 67 to 69 degrees. The maxillary dental arch width was increased by $1.9 \mathrm{~mm}$, and the crossbite of the left buccal segment was improved. Because of the space gained by the expansion, anterior maxillary teeth were lingually inclination from $123.5^{\circ}$ to $116.5^{\circ}$ in FH to U1 (Table 1, Fig. 5). Oral function improved when we removed the orthodontic devices compared to the patient's first visit and did not decrease significantly 5 years and 10 months after the treatment (Table 2).

\section{Discussion}

In cephalogram analysis, the maxillary anterior region showed a lingual inclination from $123.5^{\circ}$ to $116.5^{\circ}$ in $\mathrm{FH}$ to
U1. These results demonstrate that the quad-helix's lateral expansion performed well, and space was gained by the distal rotation of the maxillary first molar. U1 to A-pog showed a backward movement from $+6.5 \mathrm{~mm}$ to $+4.0 \mathrm{~mm}$. The mandible anterior teeth showed lingual inclination (FMIA $67.0^{\circ}$ to $69.0^{\circ}$, IMPA $90.0^{\circ}$ to $88.0^{\circ}$ ). There was a $-1.0 \mathrm{~mm}$ arch length discrepancy in the mandible dental arch. Here, when the bracket is bonded to the mandibular anterior region during the early treatment period, the anterior teeth are promoted to be inclined to the labial side, and the overjet is reduced. As a preventive measure, we bonded brackets only to the buccal segment during the early treatment period and started level- 


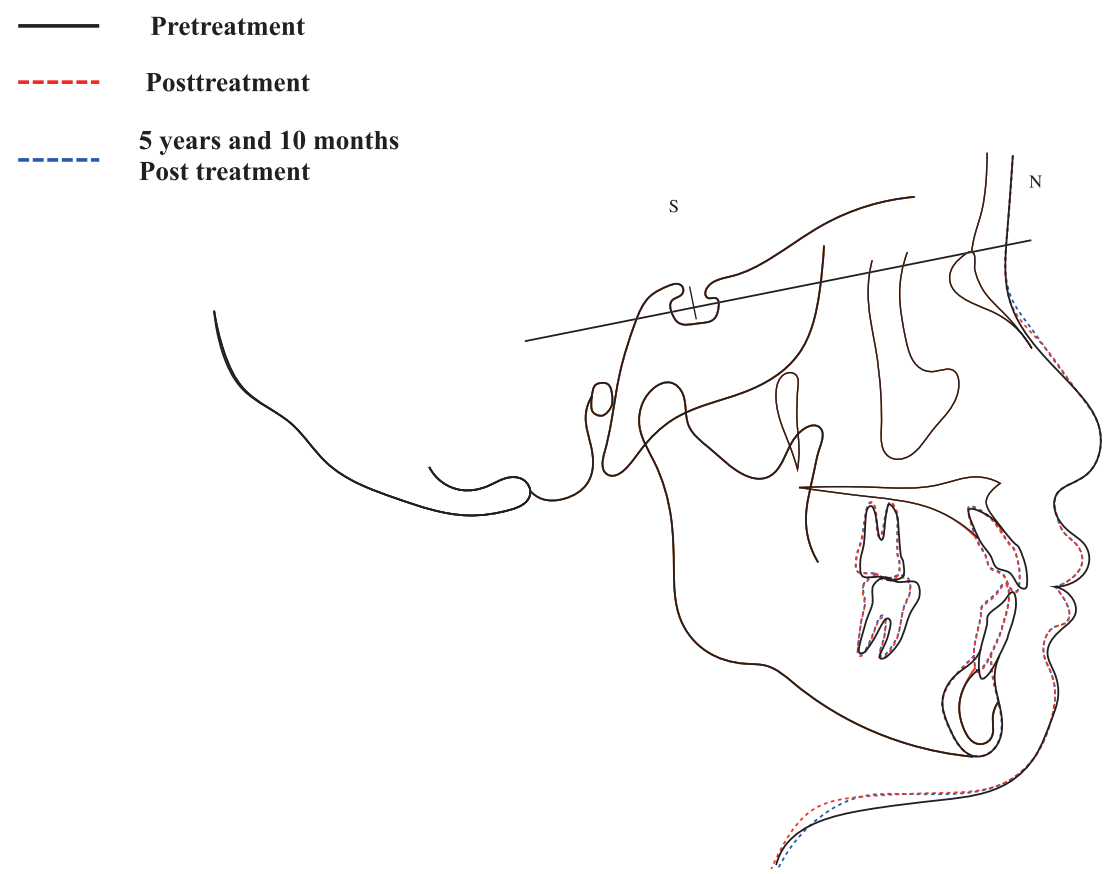

Fig. 5. Cephalometric superimposition

ing the anterior teeth after obtaining a space of $0.5 \mathrm{~mm}$ in the distal side of the mandibular canine by Class III intermaxillary elastic (The elastic force was 3.5oz). When using Class III intermaxillary elastic, the quad-helix was changed to a transpalatal arch to prevent the maxillary first molar's extrusion. There was no change in SNA, and SNB decreased by 0.5 degrees. Since the facial angle did not change, it is considered that this is due to the change in the alveolar region by the lingual side movement of the root of the mandibular anterior tooth. FMA and SNMP did not change, and therefore, their relation to vertical was maintained.

As one of the treatments of open bite cases, the intrusion of the maxillary molars promotes counterclockwise rotation of the mandible and improves the anterior occlusion(3). However, since this case was an Angle Class III and Skeletal Class III open bite, such a method increases the anterior crossbite occlusion. Therefore, we determined that the mandibular anterior teeth were extruded, and the anterior maxillary teeth were inclined to the lingual side and extrusion to improve the anterior open bite. The maxillary left third molar erupted as planned and was controlled by a multibracket. It is considered that the root paralleling is due to a bracket position. Panorama radiographs should have confirmed the direction of the root during treatment.
For myofunctional therapy, we used 'Spot' for raising the tongue's tip during swallowing and rest, 'Popping' for obtaining the sensation of presenting the whole tongue to the palate, and 'Open and close' to obtain raising of the tongue $(4,5)$. About the effectiveness of myofunctional therapy during orthodontic treatment, Jónsson et al.(6) reported that patients needed harmony of oral function for long-term stability after orthodontic treatment.

We planned to observe the long-term stability of this occlusion. In this patient, the open bite improved because of the good performance of the Class III intermaxillary elastic. However, if the desired results could not be obtained, it may be necessary to distalize the mandibular dentition with a temporary anchorage device. Five years and ten months after this treatment, the occlusion and oral functional values are stable.

\section{Conclusion}

In a Skeletal Class III case, non-extraction orthodontic treatment was successful with a multibracket device and Class III intermaxillary elastic. Long-term stable occlusion could be obtained by myofunctional therapy.

\section{Acknowledgments}

This research did not receive any specific grant from funding agencies in the public, commercial, or not-for- 
profit sectors.

\section{Conflict of Interest}

There are no conflicts of interest declare.

\section{References}

1. Vázquez-Nava F, Quezada-Castillo JA, Oviedo-Treviño S, Saldivar-González AH, Sánchez-Nuncio HR, Beltrán-Guzmán FJ, Vázquez-Rodríguez EM, Vázquez-Rodríguez CF: Association between allergic rhinitis, bottle feeding, non-nutritive sucking habits, and malocclusion in the primary dentition. Arch Dis Child, 91: 836-840, 2006.

2. Kojima K, Endo T, Shimooka S: Effects of maxillary second molar extraction on dentofacial morphology before and after anterior open-bite treatment: a cephalometric study. Odontology, 97: 43-50, 2009.

3. Deng JR, Li YA, Wang XD, Li J, Ding Y, Zhou YH: Evaluation of long-term stability of vertical control in hyperdivergent patients treated with temporary anchorage devices. Curr Med Sci, 38: 914-919, 2018.

4. Benkert KK: The effectiveness of orofacial myofunctional therapy in improving dental occlusion. Int J Orofacial Myology, 23: 35-46, 1997.

5. Smithpeter J, Covell D: Relapse of anterior open bites treated with orthodontic appliances with and without orofacial myofunctional therapy. Am J Orthod Dentofacial Orthop, 37: 605-614, 2010.

6. Jónsson T: Orofacial dysfunction, open bite, and myofunctional therapy. Eur J Orthod, 38: 235-236, 2016. 